\title{
On a Parametric Spline function
}

\author{
F.A.Abd El-Salam \\ Department of Mathematics and Engineering Physics, Faculty of Engineering, Benha University, \\ Shoubra-Cairo, Egypt
}

\begin{abstract}
This paper is concerned with the development of non-polynomial spline function approximation method to obtain numerical solution of ordinary and partial differential equations. The parametric spline function which depends on a parameter $p<0$, is discussed which reduced to the ordinary cubic spline [1] when the parameter $p=0$.
\end{abstract}

The numerical method is tested by considering an example.

Keywords : Cubic spline function, Parametric spline function, finite difference method.

\section{Introduction}

We consider a mesh $\Delta$ with nodal points $x_{i}$ on the interval $[a, b]$ such that $\Delta: a=x_{0}<x_{1}<\ldots<x_{N}=b$, where $h=x_{i}-x_{i-1}, i=1(1) N$. Assume we are given the values $\left\{y_{i}\right\}_{i=0}^{N}$ of the function $y(x)$, with $[a, b]$ as its domain of definition. A spline function of degree $\boldsymbol{m}$ with nodes at the points $x_{i}, i=1, . ., N$ is a function $s_{\Delta}(x)$ with the following properties :

(i) $s_{\Delta}(x)$ is a polynomial of degree $m$ in each subinterval $\left[x_{i}, x_{i+1}\right], i=0,1,2, \ldots, N-1$.

(ii) $s_{\Delta}(x)$ and its first $(m-1)$ derivatives are continuous on $[a, b]$.

A cubic spline function $s_{\Delta}(x)$, of class $C^{2}[a, b]$ interpolating to a function $y(x)$ defined on $[a, b]$ is such that in each interval $\left[x_{i-1}, x_{i}\right], s_{\Delta}(x)$ is a polynomial of degree at most three and the first and second derivatives of $s_{\Delta}(x)$ are continuous on $[a, b]$.

\section{Parametric Spline Function.}

Given an interval $[a, b]$ and a mesh points with knots $a=x_{0}<x_{1}<\ldots<x_{n}=b$, with $h=x_{i}-x_{i-1}, i=1,2, \ldots, N$. A function $s_{i}(x) \subset C^{2}[a, b]$ which interpolates the function $\boldsymbol{y}(\boldsymbol{x})$ at the knots $\boldsymbol{x}_{\boldsymbol{i}}$ depends on the parameter $p<0$ and reduces to a cubic spline function in the interval $\left[x_{i-1}, x_{i}\right]$ as $p=0$ is termed a parametric spline function. The parametric spline function when $p>0$ is discussed in [2]. If $s_{i}(x)$ is a parametric spline function in the interval $\left[x_{i-1}, x_{i}\right]$, then it satisfies the following differential equation:

$$
s_{i}^{\prime \prime}(x)-p^{2} s_{i}(x)=\left(M_{i-1}-p^{2} y_{i-1}\right)\left(\frac{x_{i}-x}{h}\right)+\left(M_{i}-p^{2} y_{i}\right)\left(\frac{x-x_{i-1}}{h}\right)
$$

where $M_{i}=y^{\prime \prime}\left(x_{i}\right), s_{i}\left(x_{i}\right)=y\left(x_{i}\right)$ and $\boldsymbol{p}$ is a parameter and we denote to $y\left(x_{i}\right)$ by $y_{i}$,

Solving the differential equation (1) on the interval $\left[x_{i-1}, x_{i}\right]$, subject to $s_{i}\left(x_{i}\right)=y_{i}$ and $s_{i-1}\left(x_{i-1}\right)=y_{i-1}$ we obtain:

$$
\begin{aligned}
s_{i}(x)= & \frac{h^{2}}{k^{2} \sinh k}\left\{M_{i} \sinh k z_{i-1}-M_{i-1} \sinh k z_{i}\right\} \\
& -\frac{h^{2}}{k^{2}}\left\{\left(M_{i}-w y_{i}\right) z_{i-1}-\left(M_{i-1}-w y_{i-1}\right) z_{i}\right\}
\end{aligned}
$$

where $z_{i-1}=\left(\frac{x-x_{i-1}}{h}\right), w=\frac{k^{2}}{h^{2}}$ and $k=p h$ 
The continuity of the first derivative of $s_{i}(x)$ at $\boldsymbol{x}_{i}$ in the form $s^{\prime}\left(x_{i}\right)=s_{i+1}^{\prime}\left(x_{i}\right)$ which gives

$y_{i+1}-2 y_{i}+y_{i-1}=h^{2}\left\{\alpha M_{i+1}+2 \beta M_{i}+\alpha M_{i-1}\right\}$

where

$\alpha=k^{-2}(1-k \operatorname{csch} k)$

$\beta=-k^{-2}(1-k \operatorname{coth} k)$

The consistency relation for (3) leads to equation $2 \alpha+2 \beta=1$. Which may also be expressed as $k / 2=\tan k / 2$. This equation has a zero root and an infinite number of non-zero roots. The smallest positive being $k=8.986818916$ and for $k / 2=\tan k / 2 \neq 0, \alpha+\beta=1 / 2$. For the cubic spline $\alpha=1 / 6, \beta=1 / 3$.

From equation (2) some spline relations are derived which useful in solving boundary value problems . differentiate (2) at $x_{i}, x_{i+1}$ then

$s_{i}^{\prime}\left(x_{i}\right)=-h\left(\alpha M_{i+1}+\beta M_{i}\right)+\left(\frac{y_{i+1}-y_{i}}{h}\right)$

$s_{i}^{\prime}\left(x_{i+1}\right)=h\left(\beta M_{i+1}+\alpha M_{i}\right)+\left(\frac{y_{i+1}-y_{i}}{h}\right)$

$s_{i}^{\prime}\left(x_{i}\right)+s_{i}^{\prime}\left(x_{i+1}\right)=h(\beta-\alpha)\left(M_{i+1}+M_{i}\right)+2\left(\frac{y_{i+1}-y_{i}}{h}\right)$

$s_{i}^{\prime}\left(x_{i+1}\right)+s_{i}^{\prime}\left(x_{i}\right)=h(\beta+\alpha)\left(M_{i+1}+M_{i}\right)$

when $p=0$ equation (1) take the form

$s_{i}^{\prime \prime}(x)=\left(M_{i-1}\right)\left(\frac{x_{i}-x}{h}\right)+\left(M_{i}\right)\left(\frac{x-x_{i-1}}{h}\right)$

which leads to the cubic spline function

$$
\begin{aligned}
s_{i}(x)= & \left(M_{i-1}\right) \frac{\left(x_{i}-x\right)^{3}}{6 h}+\left(M_{i}\right) \frac{\left(x-x_{i-1}\right)^{3}}{h} \\
& +\left(y_{i-1}-\frac{h^{2}}{6} M_{i-1}\right) \frac{\left(x_{i}-x\right)}{h}+\left(y_{i}-\frac{h^{2}}{6} M_{i}\right) \frac{\left(x-x_{i-1}\right)}{h} \\
& x_{i-1} \leq x \leq x_{i} .
\end{aligned}
$$

\section{Application}

(a) Numerical method for solving second-order differential equation.

Consider the second order differential equation

$$
\begin{aligned}
& y^{\prime \prime}=f(x, y), \quad a \leq x \leq b \\
& y(a)=y_{0} \\
& y(b)=y_{N}
\end{aligned}
$$

The difference equation (3) can be used to determine the approximate values of $y\left(x_{i}\right)$ at the knots points $\left\{x_{i}\right\}, i=1,2, \ldots, N$ where $N=\frac{b-a}{h}$. The difference equation when equivalent to (3) is given by

$$
y_{i+1}-2 y_{i}+y_{i-1}=\frac{h^{2}}{k^{2}}\left\{(1-k \operatorname{csch} k) f_{i+1}-2(1-k \operatorname{coth} k) f_{i}+(1-k \operatorname{csch} k) f_{i-1}\right\}
$$

where $f_{i}=f\left(x_{i}, x_{i}\right)$

Equation (15) is explicit in $y_{i+1}$ and its suitable for solving the differential equation (12)-(14). 
(b) Numerical Example.

Consider the differential equation which describe the fluid flow inside a circular cylinder in the polar form $\nabla^{2} \psi=0$

where $\quad \nabla^{2} \psi=\frac{\partial^{2} \psi}{\partial r^{2}}+\frac{1}{r} \frac{\partial \psi}{\partial r}+\frac{1}{r^{2}} \frac{\partial^{2} \psi}{\partial \theta^{2}}$

with boundary conditions
$\psi=0$,
on $r=1$
$\psi=r \sin \theta$
as $r \rightarrow \infty$
$\psi=0$
for $\theta=0, \pi$

By using the transformation $r=e^{t}$ the problem transform to

$\frac{\partial^{2} \psi}{\partial t^{2}}+\frac{\partial^{2} \psi}{\partial \theta^{2}}=0$

with boundary conditions
$\psi=0$,
on $t=0$
$\psi=e^{t} \sin \theta$
as $r \rightarrow \infty$
$\psi=0$
for $\theta=0, \pi$

by considering the parametric spline function approximation in $t$-direction with step size $h=0.2$ and mish points $t_{i}=t_{0}+i h, i=1,2, \ldots, N \quad$ In $\theta$ - direction we apply finite difference approach with step size $l=0.1 \pi$ with knots points $\theta_{j}=\theta_{0}+j l, j=1,2, \ldots, L$.and $t_{\infty}$ is taken as 0.3 . Equation (16) can be written in the form

$M_{i, j}+\frac{\psi_{i, j+1}-2 \psi_{i, j}+\psi_{i, j-1}}{l^{2}}=0$

and by using equation (3) we have the system

$$
\begin{aligned}
\psi_{i, j}= & \frac{1}{2}\left(\psi_{i+1, j}+\psi_{i-1, j}\right)-\frac{h^{2}}{2}\left\{\alpha M_{i+1, j}+2 \beta M_{i, j}+\alpha M_{i-1, j}\right\} \\
M_{i, j}= & -\frac{\psi_{i, j+1}-2 \psi_{i, j}+\psi_{i, j-1}}{l^{2}} \\
& i=1,2, \ldots, N-1, \quad j=1,2, \ldots, L-1 .
\end{aligned}
$$

From the boundary conditions we have

$$
\begin{aligned}
& M_{0, j}=0, \\
& M_{N, j}=\frac{1}{l^{2}}\left(\psi_{N, j+1}-2 \psi_{N, j}+\psi_{N, j-1}\right), \\
& \psi_{0, j}=0, \\
& \psi_{N, j}=e^{3} \sin \theta_{j}
\end{aligned}
$$

The exact solution $\psi=2 \sinh t \sin \theta$ we use Mathematica program to obtain the following numerical result with $N=6, L=10$. The computational results are present in the following table with the exact values between the brackets. This problem has earlier been discussed in [2].

\begin{tabular}{|c|c|c|c|c|c|}
\hline & $t=0.2$ & $t=0.4$ & $t=0.6$ & $t=0.8$ & $t=1.0$ \\
\hline$\theta=0.1 \pi$ & 0.125356 & 0.255717 & 0.39629 & 0.552692 & 0.731165 \\
$(0.124432)$ & $(0.253859)$ & $(0.393474)$ & $(0.54888)$ & $(0.726314)$ \\
\hline$\theta=0.2 \pi$ & 0.23844 & 0.486403 & 0.753791 & 1.5128 & 1.39067 \\
& $(0.236686)$ & $(0.485633)$ & $(0.748431)$ & $(1.4403)$ & $(1.38153)$ \\
\hline$\theta=0.3 \pi$ & 0.328185 & 0.669477 & 1.0375 & 1.44697 & 1.91421 \\
& $(0.325768)$ & $(0.664611)$ & $(1.03013)$ & $(1.43699)$ & $(1.90152)$ \\
\hline
\end{tabular}




\begin{tabular}{|c|c|c|c|c|c|}
$\theta=0.4 \pi$ & $\begin{array}{c}0.385805 \\
(0.382964)\end{array}$ & $\begin{array}{c}0.787017 \\
(0.781297)\end{array}$ & $\begin{array}{c}1.21966 \\
(1.21099)\end{array}$ & $\begin{array}{c}1.70101 \\
(1.68928)\end{array}$ & $\begin{array}{c}2.25029 \\
(2.23537)\end{array}$ \\
\hline$\theta=0.5 \pi$ & 0.405659 & 0.827519 & 1.28243 & 1.78855 & 2.3661 \\
$(0.402627)$ & $(0.821505)$ & $(1.27331)$ & $(1.77621)$ & $(2.3504)$ \\
\hline$\theta=0.6 \pi$ & 0.385805 & 0.787017 & 1.21966 & 1.70101 & 2.25029 \\
$(0.382964)$ & $(0.781297)$ & $(1.21099)$ & $(1.68928)$ & $(2.23537)$ \\
\hline$\theta=0.7 \pi$ & 0.328185 & 0.669477 & 1.0375 & 1.44697 & 1.91421 \\
& $(0.325768)$ & $(0.664611)$ & $(1.03013)$ & $(1.43699)$ & $(1.90152)$ \\
\hline$\theta=0.8 \pi$ & 0.23844 & 0.486403 & 0.753791 & 1.5128 & 1.39067 \\
$\theta=0.9 \pi$ & $0.1256686)$ & $(0.485633)$ & $(0.748431)$ & $(1.4403)$ & $(1.38153)$ \\
\hline & $(0.124432)$ & 0.255717 & 0.39629 & 0.552692 & 0.731165 \\
& $(0.253859)$ & $(0.393474)$ & $(0.54888)$ & $(0.726314)$ \\
\hline
\end{tabular}

\section{References}

[1] J.Ahlberg, E.Nilson, J.Walsh, The Theory of Splines and Their Applications, Academic Press, New York (1967).

[2] C.V.Raghavarao and S.T.P.T.Srinivas, Note on parametric spline function approximation, Computer Math. Appl. , 29(12),67-73 (1995).

[3] C.V.Raghavarao, Y.V.S.S.Sanyasiraju and S.Suresh, A note on application of cubic splines to two point boundary value problems, Computers Math. Appl.,27(11),45-48(1994).

[4] M.K.Jain and A.Tariq, Spline function approximation for differential equations, Comp.Math. in Appl. Mech. and Eng., 26,129143(1981). 